OCCURENCE OF I/29 TRANSLOCATION IN " HUNGARIAN GREY" CATTLE

\author{
A. KOVACS \\ Central Hungarian Station for Artificial Insemination \\ I440 Budapest 7o, P.O.B. 19, Hungary
}

Out of ro6 Hungarian Grey cattle 4 (3,8 p. Ioo) proved to be heterozygous carrier of $\mathrm{I} / 29$ translocation. One heifer was $60, \mathrm{XX} / 60, \mathrm{XY}$ chimaera. A simplified and effective blood culture method was used. Hypotheses concerning the origin of $\mathrm{I} / 29$ centric fusion in cattle are discussed.

\begin{abstract}
Preliminary observations on the meiotic behaviour of a $130-1 / 4^{\circ}+$ TRANSIOCATION IN DOMESTIC PIGS
\end{abstract}

\author{
W. A. KING \\ Dep. of Animal Breeding and Genetics, \\ The Swedish University of Agricultural Sciences, \\ S-750 o7 Uppsala, Sweden
}

A reciprocal translocation resulting from the exchange of almost all of chromosome number I3 and the distal end of chromosome number I 4 has previously been reported in a Swedish Yorkshive boar with approximately $52 \mathrm{p}$. Ioo reduced litter size. In an attempt to characterize the behaviour of this translocation during meiosis air-dried preparations from the testicles of heterozygous males atid cultured oocytes from heterozygous females were examined. At diakinesis/metaphase I I 7 bivalents and I quadrivalent were observed. The most common forms of the quadrivalent were a ring structure and a chain like structure. While only a limited number of second metaphase were analyzed, normal, balanced and unbalanced karyotypes were observed.

\title{
G-BANDING AND FLUORESCENT-BANDING IN SHEEP WITH HETEROZYGOUS AND HOMOZYGOUS TRANSLOCATION
}

\section{B. GLAHN. LUF'T and R. WASSMUTH \\ Institut f. Tievzucht und Haustiergenetik D-6zoo Gießen, Bismarckstraße I6}

The chromosome $I p$ - and the translocation chromosome can be identified in a ram and a ewe with heterozygous balanced translocation by G-banding and fluorescent-banding, according to the Reading Conference 1976. Based on the negative aerea in the central part and the distinct narrow telomeric band of the long arm of the autosome 1 , the chromosome I $p$-is well recognizable by the G-bande. The translocation chromosome is ordered to autosome 20 , because of three dark G-bands of similar intensity in the proximal part of this chromosome. The distal part is followed by a light segment and two dark G-bands, wich can be seen in the distal part of the chromosome i $p$.

The karyotyp of the ram with the homozygous balanced transloction, $2 n=54 \mathrm{XY}$, (I $\left.p^{-}\right)^{2}$ $t\left(\mathrm{I} p^{-} ; 2 \mathrm{O}^{+}\right)^{2}$, is well identified according to the $\mathrm{G}$-banding patterns and the RMbanding patterns.

The fluorescence staining shows a bright broad band in the distal half of the translocationchromosome. This translocationchromosome cannot be mixed up with the chromosome pairs $4-9$, because in these chromosomes exist no band of this size in the distal part of these chromosomepairs.

The G-banding technique and the fluorescence technique give an identification and the rowing of a submetazentric autosome in sheep.

The translocationchromosome is well identified based on the R-bands. A broad bright band is to be seen in the distal part of the chromosome $20^{+}$. 